ISSN 0103-5150

Fisioter. Mov., Curitiba, v. 26, n. 2, p. página 337-348, abr./jun. 2013

Licenciado sob uma Licença Creative Commons

\title{
Influência de calços na orientação postural de indivíduos com escoliose idiopática
}

\author{
Influence of the shoe lift in the postural orientation \\ in individuals with idiopathic scoliosis
}

\section{Dalva Minonroze Albuquerque Ferreira ${ }^{[a]}$, Ana Maria Forti Barela ${ }^{[b]}$, José Ângelo Barela ${ }^{[c]}$}

[a] Doutora em Ciências da Motricidade pelo Instituto de Biociências da Universidade Estadual Paulista (Unesp), Câmpus Rio Claro, SP, docente do Curso de Graduação em Fisioterapia da Faculdade de Ciências e Tecnologia Universidade Estadual Paulista (Unesp), Câmpus Presidente Prudente, SP - Brasil, e-mail: dalva@fct.unesp.br

[b] Professora titular, docente do Curso de Graduação em Educação Física e Pós-Graduação em Ciências do Movimento Humano, membro do Laboratório de Análise do Movimento, Instituto de Ciências da Atividade Física e Esporte da Universidade Cruzeiro do Sul, São Paulo, SP - Brasil, e-mail: ana.barela@cruzeirodosul.edu.br

[c] Professor Titular, docente do curso de Graduação em Educação Física e Pós-Graduação em Ciências do Movimento Humano, membro do Laboratório de Análise do Movimento, Instituto de Ciências da Atividade Física e Esporte da Universidade Cruzeiro do Sul, São Paulo, docente do Departamento de Educação Física, Instituto de Biociências da Universidade Estadual Paulista (Unesp), Câmpus Rio Claro, SP - Brasil, e-mail:jbarela@rc.unesp.br, e-mail:jose.barela@cruzeirodosul.edu.br

\section{Resumo}

Introdução: A relação entre a orientação dos segmentos e os ajustes que podem ser desencadeados por calços e palmilhas em pacientes escolióticos durante a manutenção da posição ortostática é pouco conhecida. Objetivo: Verificar alterações estáticas e associadas com mudanças unilaterais de calços na orientação postural de indivíduos com escoliose idiopática. Materiais e métodos: Grupo experimental com dez indivíduos com escoliose idiopática com curva dupla (menor $10^{\circ}$ ) e grupo controle com dez indivíduos sem escoliose (faixa etária de 13 a 24 anos). Participantes foram filmados na posição ortostática sem calço, com calço baixo $(1 \mathrm{~cm})$ e com calço alto $(3 \mathrm{~cm})$; estes foram colocados sob o pé direito e pé esquerdo dos indivíduos. Em cada condição, o participante manteve a posição estática durante 15 segundos e marcadores refletivos foram colocados em pontos anatômicos específicos. Foram calculados ângulos posturais: torácico alto; torácico médio; toracolombar e lombar e ângulos segmentares: ombro; escápula; pelve e joelho. Resultados: Na condição sem calço, diferenças foram observadas entre grupos para os ângulos posturais toracolombar e lombar e para 
o ângulo segmentar do ombro. Com calço baixo e alto, sob o pé direito, diferença foi observada entre calços para os ângulos lombar, da pelve e do joelho. Com calço baixo e alto, sob o pé esquerdo, diferença foi observada entre grupos para o ângulo toracolombar e entre calços para os ângulos da pelve e do joelho. Conclusões: A utilização de calço promove reorientação nas regiões mais baixas da coluna e nos segmentos da pelve e do joelho. Estes resultados sugerem que nas escolioses duplas, manipulação da base de apoio modifica o alinhamento do tronco que pode provocar reorganização das estruturas e busca de um novo arranjo entre segmentos em indivíduos com escoliose idiopática.

Palavras-chave: Escoliose. Postura. Manipulação. Coluna vertebral.

\section{Abstract}

Introduction: The relationship between the orientation of the segments and the adjustments that can be triggered by shoe lift and insoles in scoliotic patients during maintenance of standing position is unclear.

Objective: To verify static changes and those associated with unilateral manipulations of shoe lift in the postural orientation in people with idiopathic scoliosis. Materials and methods: Experimental group composed of ten patients with idiopathic scoliosis with double curve (less $10^{\circ}$ ) and control group with ten participants without scoliosis (aging from 13 to 24 years). Participants were videotaped in upright stance, standing with no, low $(1 \mathrm{~cm})$, and high $(3 \mathrm{~cm})$ shoe lift, which were placed under right and left shoe. In each condition, the participant maintain upright stance for 15 seconds and reflective markers were affixed on specific anatomical places. Postural angles were obtained: high thoracic; medium thoracic; thoracolumbar; and lumbar, as well segmental angles: shoulder; scapula; pelvis; and knee. Results: In the no shoe lift condition, differences were observed between groups for high and medium thoracic angles and for shoulder. With low and high shoe lift under the right foot, difference was observed between shoe lift heights for high thoracic, for pelvis and knee angles. With low and high shoe lift under the left foot, differences between groups were observed for thoracolumbar angle and between shoe lift heights for pelvis and knee angles. Conclusions: The shoe lift promotes reorientation in the lower regions of the spine and segments of pelvis and knee. It might be suggested that in the scoliosis with double curves, manipulation in the basis of support changes the alignment of the trunk that might promote structural reorganization and the search of new adjustments among segments in individuals with idiopathic scoliosis.

Keywords: Scoliosis. Posture. Manipulation. Spine.

\section{Introdução}

A manutenção da postura requer controle e informação sensorial contínua sobre a orientação corporal e as forças que atuam sobre cabeça, tronco, cintura pélvica e membros inferiores (1). Dentre os muitos segmentos do corpo que influenciam a orientação postural, o tronco tem papel fundamental, principalmente por ser o esqueleto axial do corpo humano; porém, esse segmento tem recebido pouca atenção dos estudiosos. Investigações mais detalhadas sobre o papel da orientação do tronco e da coluna vertebral durante a manutenção da postura podem ampliar a compreensão do controle corporal como um todo, além de possibilitar a detecção de possíveis causas e o entendimento da progressão de deformidades do tronco ou da coluna vertebral, como por exemplo, na escoliose idiopática $(2,3)$.

A escoliose é uma deformidade considerada tridimensional, uma vez que altera a coluna vertebral através da lordose no plano sagital, um desvio lateral no plano frontal e uma rotação no plano transversal (4-8). Em aproximadamente 20\% dos casos de escoliose, a causa inicial é conhecida; no restante, as causas estão provavelmente relacionadas a hábitos posturais, sendo denominadas de escolioses idiopáticas (9).

Apesar da incidência elevada - de 1 a 13\% da população - e das consequências da escoliose tais como dor, redução da função pulmonar, lesões degenerativas, retrações e espasmos musculares, não há muita informação disponível sobre a relação entre a 
orientação dos segmentos e os possívies ajustes desencadeados por manipulações como calços e palmilhas em pacientes escolióticos durante a manutenção da posição ortostática.

Na manutenção da posição ortostática, ajustes posturais são necessários e podem ser acompanhados de uma grande variedade de respostas nas articulações dos tornozelos, joelhos e coluna vertebral, de forma independente ou combinada (10). Alguns estudos relacionaram a etiologia da escoliose a distúrbios do equilíbrio estático e dinâmico (11-14). Ainda, alterações posturais da coluna vertebral podem desencadear respostas e reorganização de segmentos corporais que influenciam a orientação dos segmentos e posicionamento do tronco.

Uma escoliose funcional resultante de um desequilíbrio postural decorente de dor, espasmos musculares ou outros fatores pode progredir para uma escoliose estrutural se os fatores incitantes não forem identificados e corrigidos (15). Um modelo de "ciclo vicioso" prediz que uma carga assimétrica contínua, mesmo que pequena, pode provocar progressão da curvatura, exceto nos casos em que tal ação seja revertida. No entanto, mesmo com uma deformidade estrutural severa, se o estado de carga contínua for revertido e a pressão assimétrica sobre o platô em crescimento for restaurada, a curvatura pode ser revertida. Ainda, conquanto a deformidade estrutural possa afetar apenas o ápice da curva, outras partes da coluna permanecem flexíveis e podem ser corrigidas pela mudança na postura (15). No caso de deformidade vertebral acompanhada de discrepância do comprimento dos membros, a magnitude da curva pode ser reduzida em até 20 graus $(15,16)$, embora não seja corrigida pelo uso de palmilhas (16). Por outro lado, nos estágios iniciais de escoliose associada à discrepância no comprimento dos membros inferiores, as anomalias das curvaturas da coluna podem ser corrigidas com o uso de palmilha sob o pé para reduzir a discrepância do comprimento dos membros que gera assimetria postural (15).

A utilização da palmilha pode ser entendida como um calço mecânico, e também como um recurso para aumentar a estimulação sensorial de regiões específicas dos pés $(15,16)$. A região plantar possui uma variedade de receptores sensoriais que são sensíveis às variações de deformação. Em resposta aos estímulos sensoriais, o sistema nervoso central regula a tensão dos músculos posturais e regula as oscilações do corpo por intermédio dos músculos extrínsecos e intrínsecos dos pés (2). Alguns estudos têm sugerido que bases de suporte (foot template) (17), palmilhas (shoe lift) $(15,16,18,19)$, elevação dos calcanhares (hitch exercise) (20), ou calços (heel lift) (21) devem ser utilizadas para produzir estímulos na região plantar. As adaptações posturais agudas, decorrentes da manipulação no apoio plantar, podem ter um impacto sobre a geometria vertebral; se essas reações posturais persistirem ou um processo de adaptação ocorrer, possíveis efeitos secundários podem ser desenvolvidos e potenciais impactos sobre a saúde dos pacientes escolióticos devem ser considerados (18).

Especificamente, o objetivo mecânico referente à utilização de bases de suporte, palmilhas ou calços para tratamento da escoliose é alinhar de forma ascendente os pés, joelhos, pelves, escápulas, ombros e coluna vertebral, de forma a ajustar a orientação do corpo no espaço. Esses ajustes podem impedir a evolução e a compensação dos desvios posturais e, por causa do alinhamento, evitar agravamentos de lesões estruturais degenerativas, dor, espasmos e retrações musculares.

Apesar de o uso de calço ser eficaz para o realinhamento postural de indivíduos com escoliose, até o momento, nenhum estudo examinou os efeitos agudos da utilização de calços de diferentes alturas para o alinhamento postural. Sendo assim, o presente estudo avaliou as alterações estáticas agudas na coluna vertebral e nos segmentos corporais durante a posição ortostática e os possíveis efeitos do uso de calços na reorientação postural de pacientes com escoliose idiopática.

\section{Metodologia}

\section{Participantes}

Vinte indivíduos encaminhados por médicos ortopedistas de Presidente Prudente e oriundos de triagem realizada no Centro de Estudos e Atendimentos em Fisioterapia e Reabilitação (Ceafir) da Faculdade de Ciências e Tecnologia, Unesp/Presidente Prudente participaram deste estudo. Inicialmente, os potenciais participantes foram avaliados por meio do Teste de Adams (22) (flexão anterior da coluna vertebral) para verificar e medir a gibosidade. Participantes com gibosidade maior que $5 \mathrm{~mm}$, foram convidados a realizar um exame radiográfico para confirmar ou não a presença de escoliose estrutural idiopática por meio 
da medida do ângulo de Cobb (8). Os participantes sem gibosidade ou com gibosidade mínima $(<5 \mathrm{~mm})$ compuseram o grupo controle $(\mathrm{n}=10)$, sendo todos do gênero feminino, e classificados sem escoliose. Os participantes com ângulo de Cobb $\geq 10$ graus compuseram o grupo experimental $(n=10)$, nove participantes eram do gênero feminino e um do gênero masculino; todos foram classificados com escoliose idiopática. As informações sobre idade e medidas antropométricas dos dois grupos são apresentadas na Tabela 1. Ainda, os participantes do grupo experimental apresentavam dupla curva: uma curva torácica (média do ângulo de Cobb $=21,7^{\circ} \pm 9,86^{\circ}$, intervalo de $10^{\circ}$ a $38^{\circ}$ ) e uma curva lombar (média do ângulo de Cobb $=19,5^{\circ} \pm 8,08^{\circ}$, intervalo de $10^{\circ}$ a $31^{\circ}$ ).

Após serem informados sobre os objetivos e procedimentos do estudo, os participantes ou os responsáveis legais assinaram o Termo de Consentimento Livre e Esclarecido para participação no presente estudo. Esse termo e os procedimentos foram aprovados pelo Comitê de Ética em Pesquisa do Instituto de Biociências, Unesp/Rio Claro (parecer CEP 109/2007, protocolo 0522).

\section{Procedimentos}

Os participantes compareceram ao Laboratório de Fisioterapia Aplicada ao Movimento Humano, Faculdade de Ciências e Tecnologia, Unesp/Presidente Prudente e foram preparados para realização do experimento. Para tanto, todos os participantes permaneceram descalços e em traje de banho, e um dos experimentadores devidamente treinados afixou marcadores refletivos em pontos anatômicos específicos, que foram definidos com base em estudos anteriores (23-27). Sendo assim, 14 marcadores (13 mm de diâmetro) foram afixados nos seguintes pontos anatômicos de cada participante: processo espinhoso da sétima vértebra cervical (C7), segunda (T2), quarta
(T4), sexta (T6), oitava (T8), décima (T10) e décima segunda (T12) vértebras torácicas, segunda (L2) e quarta (L4) vértebras lombares e na crista sacral medial (S1), ângulos inferiores das escápulas direita (AIED) e esquerda (AIEE), e crista ilíaca posterossuperior direita (CIPSD) e esquerda (CIPSE). Ainda, quatro marcadores (25 mm de diâmetro) foram afixados no acrômio direito (AD) e esquerdo (AE), e na linha articular da fossa poplítea direita (FPD) e esquerda (FPE).

Em seguida, os participantes assumiram a posição conhecida como "posição nominal dos pés" $(28,29)$, mantendo, portando, os pés separados na largura do quadril; foi definida uma linha média em cada pé conectando a região medial do calcanhar com o segundo artelho. A partir dessa linha, os pés ficaram rodados externamente em $16^{\circ}$, e essa posição foi reproduzida sobre uma folha com o desenho da impressão plantar. Os membros superiores ficaram relaxados ao lado do corpo, e os participantes foram orientados a manter a cabeça na linha média, sem qualquer ajuste do avaliador durante as coletas.

Os participantes foram filmados por três câmeras previamente ajustadas e colocadas em suportes específicos fixados na parede, com holofotes colocados acima e atrás de cada câmera. Uma câmera foi posicionada perpendicularmente e atrás do participante, e as outras duas câmeras foram posicionadas nas laterais direita e esquerda da câmera central, formando um ângulo de aproximadamente 60 graus em ralação a essa câmera. As imagens capturadas pelas filmadoras foram sincronizadas por meio um dispositivo luminoso, visível pelas três câmeras, que era acionado manualmente por um dos experimentadores no início de cada tentativa realizada pelo participante.

Cada participante realizou cinco tentativas, permanecendo na posição ortostática durante 15 segundos em cada uma. A primeira tentativa foi realizada com o participante assumindo a "postura nominal"; a segunda e terceira tentativas foram realizadas com o participante apoiando ora pé direito, ora pé esquerdo

Tabela 1 - Valores das médias ( \pm desvio padrão) da idade, massa, estatura e IMC (Índice de Massa Corpórea) dos grupos controle $(n=10)$ e experimental $(n=10)$

\begin{tabular}{llllc}
\hline Grupo & Idade (anos) & Massa $(\mathbf{k g})$ & Estatura $\mathbf{k g} / \mathbf{m}^{2}$ & IMC \\
\hline Controle & $19,5( \pm 1,03)$ & $53,9( \pm 13,10)$ & $1.64( \pm 0,08)$ & $20,7( \pm 2,74)$ \\
Experimental & $20,0( \pm 2,88)$ & $56,6( \pm 9,29)$ & $1,66( \pm 0,09)$ & $19,7( \pm 2,97)$ \\
\hline
\end{tabular}

Fonte: Dados da pesquisa. 
sobre um calço de madeira, $35 \mathrm{~cm} \times 20 \mathrm{~cm} \times 1 \mathrm{~cm}$ (largura $\times$ comprimento $\times$ altura) - "calço baixo"; e as duas últimas tentativas foram realizadas com o participante apoiando ora pé direito, ora pé esquerdo sobre um calço de $3 \mathrm{~cm}$ de altura - "calço alto". Essas alturas foram selecionadas porque $1,5 \mathrm{~cm}$ é considerado uma diferença real do comprimento dos membros inferiores, podendo gerar escoliose estrutural não idiopática (30). 0 calço de $3 \mathrm{~cm}$, por outro lado, constitui uma manipulação extrema para uma possível adaptação em longo prazo, com realinhamento postural, podendo auxiliar na prescrição clínica destes como complemento no tratamento das escolioses idiopáticas. A ordem de colocação do calço sob o pé direito ou esquerdo foi definida por sorteio e um breve intervalo de 1 a 2 minutos entre as tentativas ocorreu, para minimizar qualquer efeito de fadiga muscular.

\section{Tratamento dos dados}

Os dados da filmagem de todos os participantes correspondentes aos 5 segundos intermediários de cada tentativa foram transferidos para um computador via placa de captura, com a utilização do programa Ariel Performance Analysis System - APAS (Ariel Dynamics, Inc.). Esses dados foram digitalizados automaticamente com o mesmo programa para obtenção das coordenadas " $x$ ", " $y$ " e " $z$ " dos marcadores que foram afixados nos pontos anatômicos dos participantes. 0 procedimento para a transformação das coordenadas reais dos dados adquiridos foi o da transformação linear direta (direct linear transformation - DLT). 0 procedimento DLT para esses dados utilizou um sistema de referência com 12 pontos controle e volume de 1,07 ×1,66 $\times$ $1,23 \mathrm{~m}^{3}$ (comprimento $\times$ altura $\times$ profundidade). $\mathrm{Em}$ seguida, os dados foram filtrados utilizando um filtro digital Butterworth, passa-baixa, com frequência de corte de $5 \mathrm{~Hz}$, definida a partir da análise de resíduo (31) e os ângulos relativos e absolutos foram calculados conforme descrito adiante.

Para este estudo, especificamente, quatro ângulos relativos com base no plano frontal foram calculados conforme convenção utilizada em estudos prévios $(23,24,32)$ com o objetivo de avaliar as modificações posturais da coluna vertebral. Sendo assim, os dados dos marcadores afixados em T2-T4 e T4-T6 formaram o ângulo "torácico alto" ( $\alpha 1)$, em T6-T8 e T8-T10 formaram o ângulo "torácico médio $(\alpha 2)$, em T10-T12 e T12-L2 formaram o ângulo "toracolombar" ( $\alpha 3$ ), e em L2-L4 e L4-S1 formaram o ângulo "lombar" ( $\alpha 4)$ (Figura 1A). Ainda, quatro ângulos absolutos com base no plano frontal foram calculados com o intuito de examinar o alinhamento do tronco em relação à horizontal, conforme convenção utilizada em estudos anteriores $(16,17,27,33-36)$. Sendo assim, os dados dos marcadores afixados em AE-AD formaram o ângulo referente ao alinhamento dos ombros direito e esquerdo ( $\beta 1$ ); em EE-ED formaram o ângulo referente ao alinhamento das escápulas direita e esquerda $(\beta 2)$; em CIPSE-CIPSD formaram o ângulo referente ao alinhamento da pelve ( $\beta 3$ ); e em FPE-FPD formaram o ângulo referente ao alinhamento dos joelhos direito e esquerdo ( $\beta 4$ ) (Figura 1B).

Os ângulos posturais e segmentares foram calculados para cada uma das condições experimentais. De acordo com a convenção utilizada, valores angulares menores que 180 graus indicaram curvatura à esquerda e valores angulares maiores que 180 graus indicaram curvatura à direita. Finalmente, esses ângulos foram subtraídos de um valor referência ângulo basal de $180^{\circ}$ - e transformados em valores absolutos, sendo posteriormente calculada a média para cada participante e condição.

\section{Análises estatísticas}

Duas análises de multivariância (MANOVAs) foram realizadas, tendo como fator o grupo (controle e experimental) e como variáveis dependentes os valores dos ângulos posturais $(\alpha 1,2, \alpha 3$ e $\alpha 4)$ para a primeira MANOVA, e os valores dos ângulos segmentares $(\beta 1, \beta 2, \beta 3$ e $\beta 4)$ para a segunda MANOVA, obtidos na condição sem calço. Duas outras MANOVAs foram realizadas, tendo como fatores grupo, condição ("calço baixo" e "calço alto") e pé (direito), sendo esse último fator tratado como medidas repetidas. As variáveis dependentes foram os valores dos ângulos relativos e os valores dos ângulos absolutos, para a primeira e a segunda MANOVA respectivamente. Finalmente, outras duas MANOVAs foram realizadas, tendo os dois primeiros fatores e as mesmas variáveis dependentes, porém o fator pé foi diferente (esquerdo).

Todas as análises foram realizadas utilizando o software estatístico SPSS (versão 10.0). Quando necessário, análises univariadas foram realizadas e testes post-hoc de Tukey com ajuste de Bonferroni foram utilizados. Finalmente, o nível de significância foi mantido em 0,05 em todas as análises. 

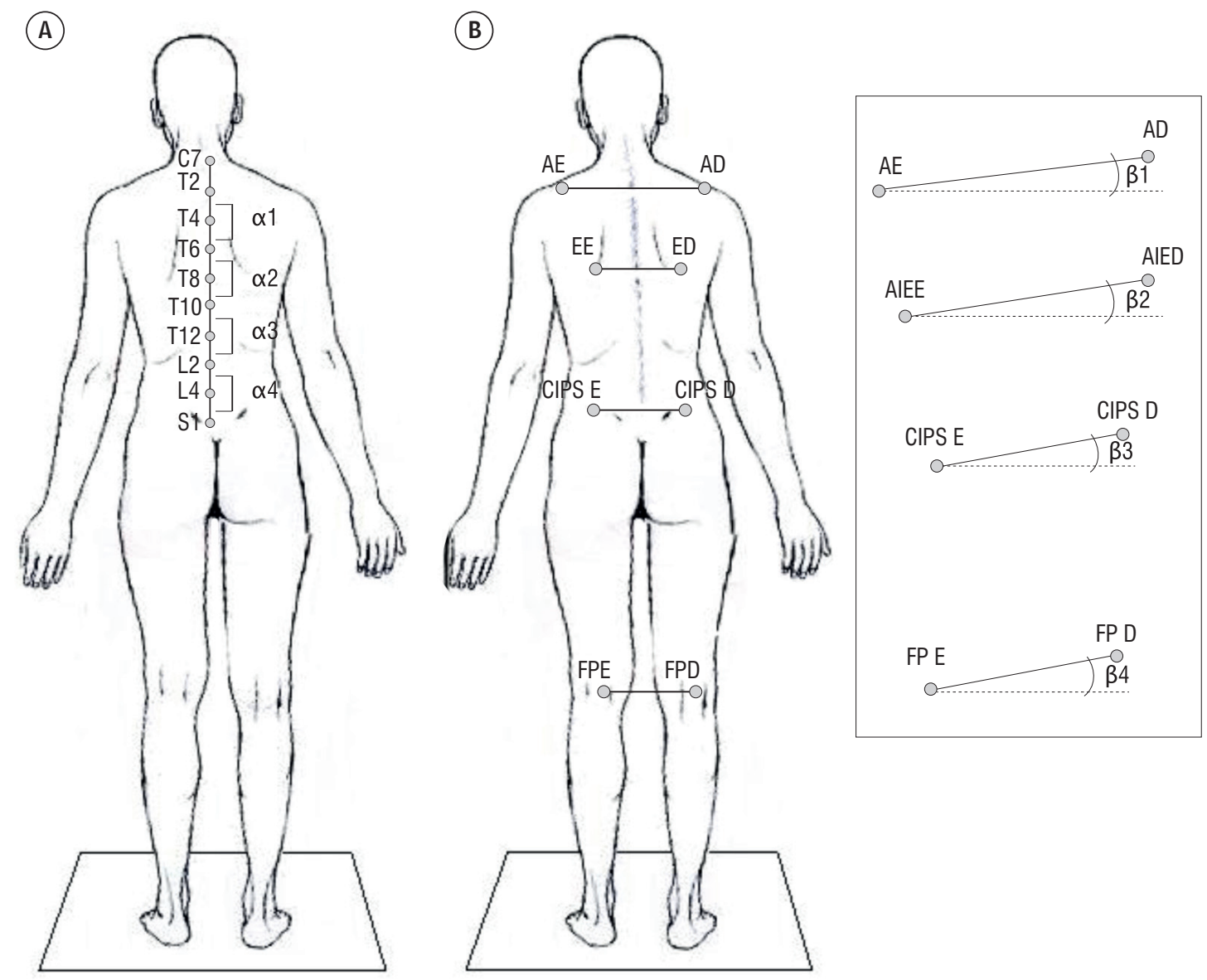

Figura 1 - Ângulos posturais da coluna vertebral no plano frontal (A) e ângulos segmentares em relação ao eixo $x$

Legenda: $\mathrm{A}-\alpha 1$ = ângulo torácico alto, $\alpha 2$ = ângulo torácico médio, $\alpha 3=$ ângulo toracolombar, $\alpha 4=$ ângulo lombar; $B-\alpha 1=$ ângulo do ombro, $\alpha 2$ = ângulo da escápula, $\alpha 3$ = ângulo da pelve, $\alpha 4$ = ângulo do joelho.

Fonte: Dados da pesquisa.

Nota: Em A, apresentam-se ângulo torácico alto (T2T4-T4T6), ângulo torácico médio (T6T8-T8T10), ângulo toracolombar (T10T12-T12L2) e ângulo lombar (L2L4-L4S1); em B, apresentam-se ângulo do ombro, AD (acrômio direito) e AE (acrômio esquerdo), ângulo da escápula, AIED (ângulo inferior da escápula direita) e AIEE (ângulo inferior da escápula esquerda), ângulo da pelve CIPSD (crista ilíaca posterossuperior direita) e CIPSE (crista ilíaca posterossuperior esquerda) e ângulo do joelho FPD (linha articular da fossa poplítea direita) e FPE (linha articular da fossa poplítea esquerda).

\section{Resultados}

A Figura 2 apresenta os valores médios dos ângulos posturais $(\alpha 1, \alpha 2, \alpha 3$ e $\alpha 4)$ e segmentares $(\beta 1, \beta 2$, $\beta 3$ e $\beta 4$ ) para os grupos controle e experimental, na condição sem calço. MANOVA, envolvendo os ângulos segmentares, indicou diferença entre os grupos, Wilks' Lambda $=0,363, \mathrm{~F}(4,15)=6,586, \mathrm{p}<0,005$. Análises univariadas apontaram diferenças para os ângulos toracolombar ( $\alpha 3), \mathrm{F}(1,18)=9,181, \mathrm{p}<0,01$, e lombar $(\alpha 4), F(1,18)=4,497, p<0,05$, sendo que em ambos os casos os ângulos observados para os participantes do grupo experimental foram maiores do que oos observados para os participantes do grupo controle. MANOVA, envolvendo os ângulos segmentares, também indicou diferença entre os grupos, Wilks' Lambda $=0,515, F(4,15)=3,53, p<0,05$. Análises univariadas indicaram diferenças apenas para o ângulo do ombro $(\beta 1), F(1,18)=14,163, p<0,005$, sendo que o ângulo observado para os participantes 
do grupo experimental foi maior que o observado para os participantes do grupo controle.

A Figura 3 apresenta os valores médios dos ângulos posturais $(\alpha 1, \alpha 2, \alpha 3$ e $\alpha 4)$ para os grupos controle e experimental, na condição com calço direito baixo (A) e calço direito alto (B). MANOVA, envolvendo os ângulos posturais, indicou diferença entre os grupos, Wilks' Lambda $=0,550, \mathrm{~F}(4,15)=3,072$, $\mathrm{p}<$ 0,05 . Análises univariadas, entretanto, não apontaram qualquer diferença, apenas marginalidade para o ângulo lombar $(\alpha 4), F(1,18)=3,866, p=0,065$, sugerindo que o ângulo lombar observado para os participantes do grupo experimental apresentou tendência a ser maior que o observado para os participantes do grupo controle. MANOVA também indicou diferença entre as condições de calço baixo e alto, Wilks' Lambda $=0,485, \mathrm{~F}(4,15)=3,989, \mathrm{p}<0,05$. Análises univariadas apontaram diferenças apenas para o ângulo lombar $(\alpha 4), F(1,18)=6,019, p<0,05$, sendo que o mesmo foi menor na condição de calço baixo do que na condição de calço alto.

A Figura 3 também apresenta os valores médios dos ângulos segmentares $(\beta 1, \beta 2, \beta 3$ e $\beta 4)$ para os grupos controle e experimental na condição com calço direito baixo (C) e calço direito alto (D). MANOVA indicou diferença apenas entre as condições de calço baixo e alto, Wilks' Lambda = 0,024, F $(4,15)=154,459$, $\mathrm{p}<0,001$. Análises univariadas indicaram diferenças para os ângulos da pelve $(\beta 3), \mathrm{F}(1,18)=20,614, \mathrm{p}<$ 0,001 , e do joelho $(\beta 4), F(1,18)=584,2,(p<0,001)$, sendo que para ambos os casos, os ângulos observados para a condição de calço baixo foram menores do que os observados para a condição de calço alto.

A Figura 4 apresenta os valores médios dos ângulos posturais ( $\alpha 1, \alpha 2, \alpha 3$ e $\alpha 4)$ para os grupos controle e experimental na condição com calço esquerdo baixo (A) e calço esquerdo alto (B). MANOVA indicou diferença apenas entre os grupos, Wilks' Lambda = $0,349, \mathrm{~F}(4,15)=6,98, \mathrm{p}<0,005$. Análises univariadas apontaram diferenças apenas para o ângulo toracolombar ( $\alpha 3), F(1,18)=9,278, p<0,01$, sendo que o ângulo toracolombar observado para os participantes do grupo experimental foi maior do que o observado para os participantes do grupo controle.

Na Figura 4 apresentam-se também os valores médios dos ângulos segmentares $(1, \beta 2, \beta 3$ e $\beta 4)$ para os grupos controle e experimental na condição com calço esquerdo baixo (C) e calço esquerdo alto (D). MANOVA indicou diferença apenas entre as condições de calço baixo e alto, Wilks' Lambda $=0,032, \mathrm{~F}(4,15)$ $=113,68, p<0,001$. Análises univariadas indicaram diferenças para os ângulos da pelve $(\beta 3), \mathrm{F}(1,18)=$ $97,933, p<0,001$, e do joelho $(\beta 4), F(1,18)=482,696$, $\mathrm{p}<0,001$, sendo que para ambos os casos os ângulos na condição de calço baixo foram menores do que os observados para a condição de calço alto.

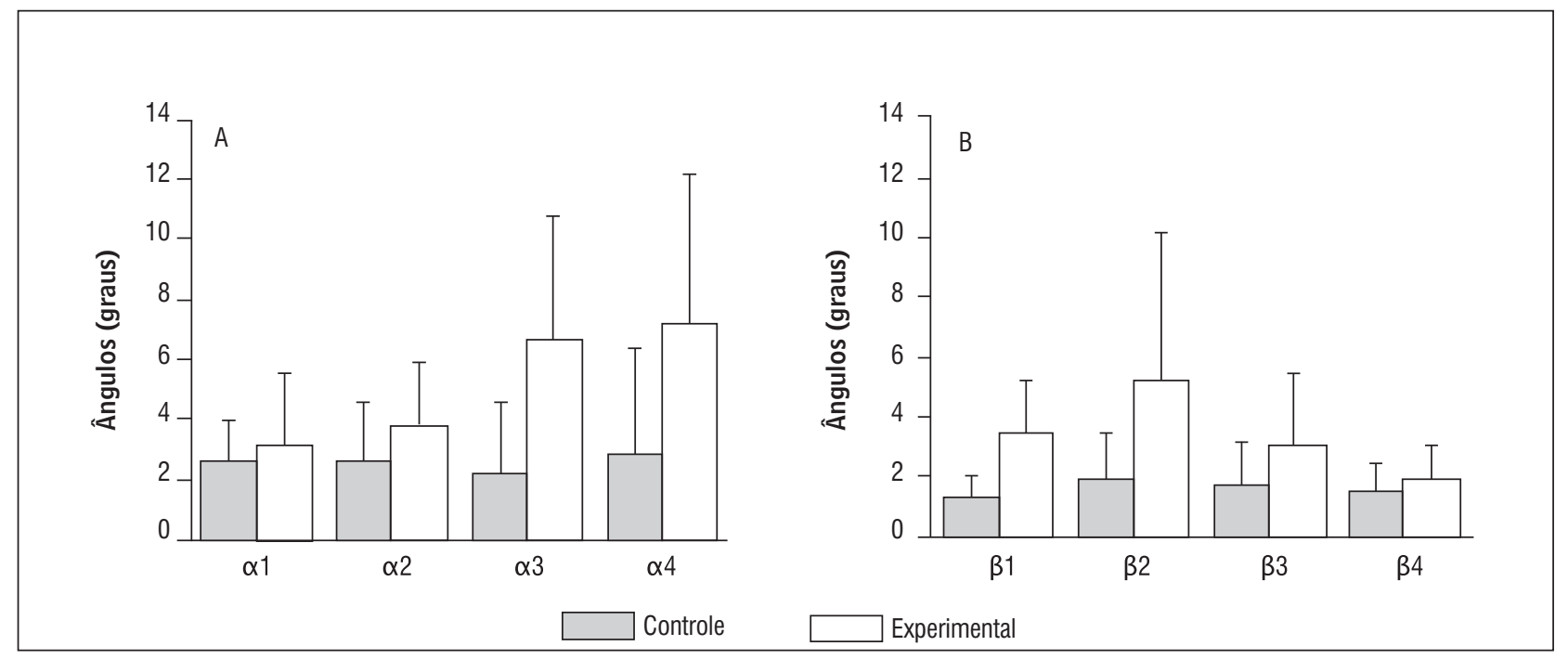

Figura 2 - Médias e desvios padrão dos ângulos posturais (A) e dos ângulos segmentares (B) para os grupos controle e experimental, na condição sem calço, na tarefa estática

Legenda: $\mathrm{A}-\alpha 1$ = ângulo torácico alto, $\alpha 2$ = ângulo torácico médio, $\alpha 3=$ ângulo toracolombar, $\alpha 4=$ ângulo lombar; $\mathrm{B}-\beta 1=$ ângulo do ombro, $\beta 2$ = ângulo da escápula, $\beta 3$ = ângulo da pelve, $\beta 4$ = ângulo do joelho.

Fonte: Dados da pesquisa. 

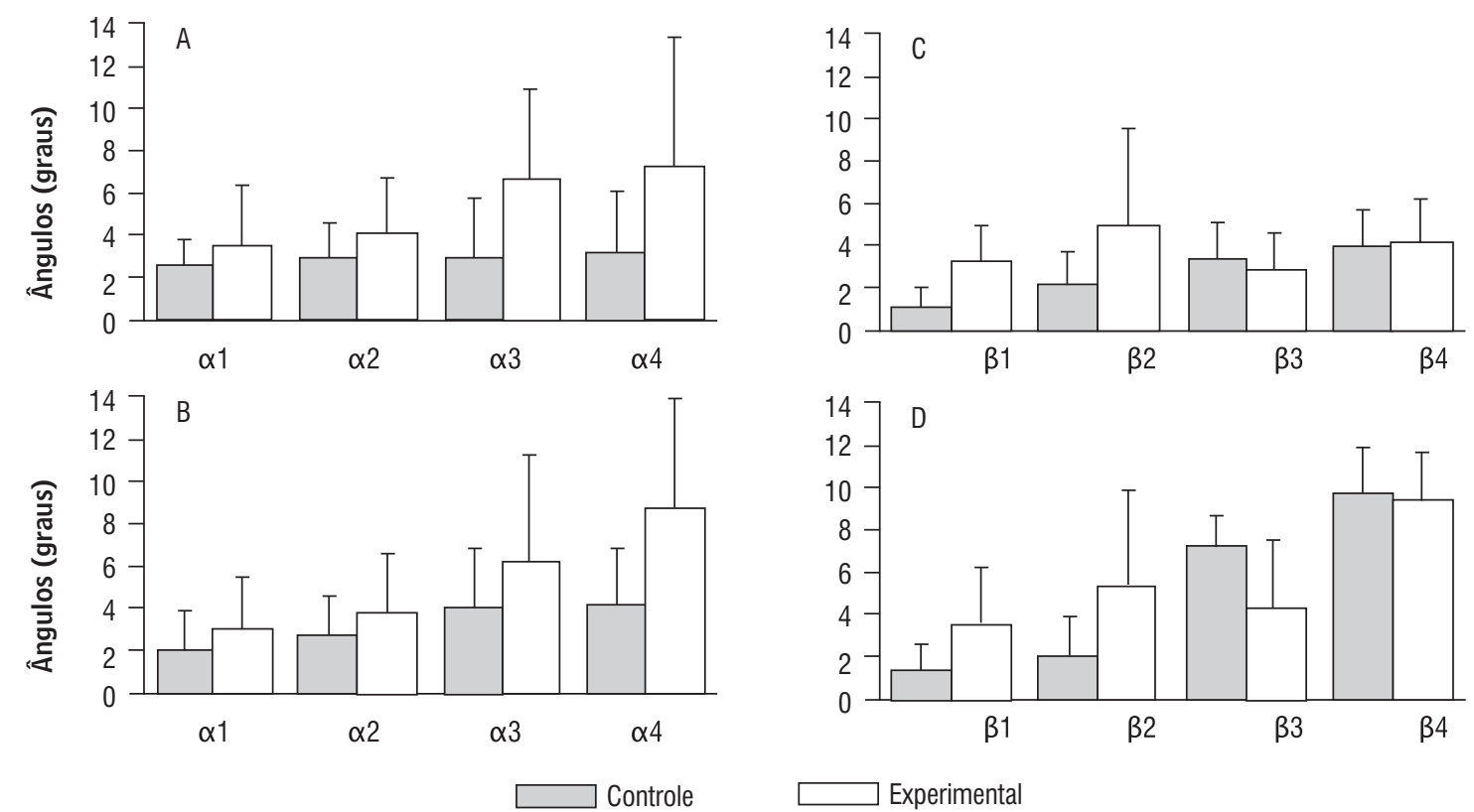

Figura 3 - Médias e desvios padrão dos ângulos posturais com calço direito baixo (A) e com calço direito alto (B) e dos ângulos segmentares com calço direito baixo (C) e com calço direito alto (D) para os grupos controle e experimental na tarefa estática Legenda: $\mathrm{A}-\alpha 1$ = ângulo torácico alto, $\alpha 2=$ ângulo torácico médio, $\alpha 3=$ ângulo toracolombar, $\alpha 4=$ ângulo lombar; $\mathrm{B}-\beta 1=$ ângulo do ombro, $\beta 2$ = ângulo da escápula, $\beta 3$ = ângulo da pelve, $\beta 4$ = ângulo do joelho.

Fonte: Dados da pesquisa.
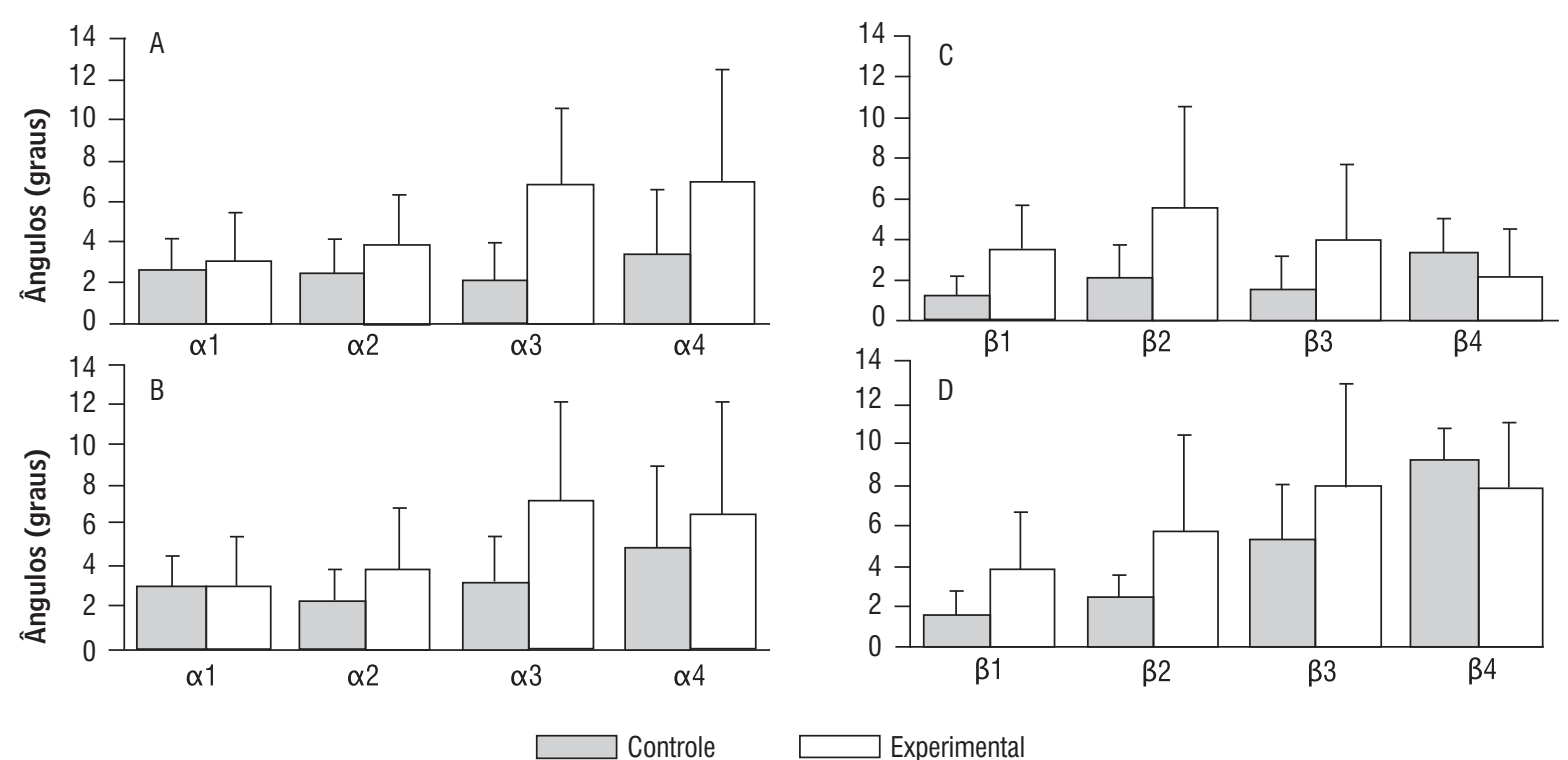

Figura 4 - Médias e desvios padrão dos ângulos posturais com calço esquerdo baixo (A) e com calço esquerdo alto (B) e dos ângulos segmentares beta com calço esquerdo baixo (C) e com calço alto (D) para os grupos controle e experimental na tarefa estática

Legenda: $\mathrm{A}-\alpha 1$ = ângulo torácico alto, $\alpha 2=$ ângulo torácico médio, $\alpha 3=$ ângulo toracolombar, $\alpha 4=$ ângulo lombar; $\mathrm{B}-\beta 1=$ ângulo do ombro, $\beta 2$ = ângulo da escápula, $\beta 3$ = ângulo da pelve, $\beta 4$ = ângulo do joelho.

Fonte: Dados da pesquisa. 


\section{Discussão}

O objetivo do presente estudo foi avaliar as alterações estáticas na coluna vertebral de pacientes com escoliose idiopática na posição ortostática e nas mudanças unilaterais de calços baixo e alto. Os resultados indicaram que embora as curvas duplas nos pacientes com escoliose afetem toda a coluna, as regiões mais afetadas são a toracolombar e lombar, para os ângulos posturais, e o alinhamento do ombro como evidenciado pelos ângulos segmentares. Esses resultados da análise na posição estática sem calço demonstram que, mesmo nas escolioses duplas, consideradas compensadas, estes ângulos ainda refletem uma assimetria importante no tronco de pacientes com escoliose; portanto, essas medidas também podem ser confiáveis para comparações entre grupos.

De forma geral, pacientes com escoliose em posição estática apresentaram, no presente estudo, alteração na orientação da coluna na altura das estruturas toracolombar e lombar, apresentando maior curvatura. Essa maior curvatura nessas regiões induz reorientação dos segmentos do tronco como um todo que acabam refletindo em maior inclinação do ombro. Essas observações e os respectivos valores dos ângulos mensurados para a posição estática correspondem aos observados anteriormente $(37,38)$. Diferenças maiores que $7^{\circ}$ nos ângulos posturais, principalmente em curvas baixas (toracolombar e lombar), apontam assimetrias importantes na coluna vertebral e devem ser consideradas relevantes, pois podem refletir com maior segurança a assimetria provocada pela escoliose $(39,40,41)$.

As reorientações posturais observadas em pacientes com escoliose idiopática também interferem na manutenção do equilíbrio, com estes apresentando maior oscilação corporal, pelos valores do desvio padrão, durante a manutenção da postura ereta $(17,42$, $43,44)$. Apesar de resultados contraditórios observados em alguns estudos $(45,46,47)$, há indicativos de que quanto maiores as curvaturas toracolombar e lombar, maior é a instabilidade postural $(12,42)$; ainda que pacientes com curvas duplas aparentemente compensem melhor a reorientação postural apresentando diferenças menores na manutenção do controle postural (12).

Os valores dos ângulos segmentares observados no presente estudo também corresponderam aos observados em estudos anteriores $(17,35)$. Especificamente, Nault et al. (17), comparando pacientes com escoliose e indivíduos do grupo controle, observaram diferenças significativas do ângulo formado pelos acrômios no plano frontal; encontraram também média similar do ângulo segmentar do ombro e encontraram diferença no ângulo da pelve, similar ao ângulo segmentar da pelve. Dessa forma, a análise da orientação segmentar utilizada no presente estudo também mostrou-se apropriada e importante para indicar possíveis alterações de reorientação de segmentos corporais decorrentes da escoliose idiopática. Ainda, a observação de alteração nas curvas da coluna, em específico nas regiões toracolombar e lombar, é importante não apenas para diagnosticar o paciente escoliótico, mas também para prognosticar possíveis alterações funcionais como parâmetros de evolução para o tratamento utilizado, pois refletiram melhor a diferença em relação ao grupo controle.

Um segundo aspecto do objetivo do presente estudo foi verificar possíveis alterações na coluna vertebral de pacientes com escoliose idiopática provocadas pelo uso de diferentes calços posicionados unilateralmente sob a base de apoio. Os resultados observados indicaram que os calços, baixo e alto, provocaram alterações apenas na região lombar. As curvas maiores eram as curvas torácicas, com mais chances, consequentemente, de serem as curvas principais, menos flexíveis em relação às curvas secundárias, mais afetadas pela deformidade no ápice da curva e, portanto, menos suscetíveis a alterações (16). Por outro lado, os maiores efeitos dos calços seriam esperados nas curvas mais baixas (21), por serem as curvas secundárias ou compensatórias mais flexíveis (15). Portanto, os resultados observados corroboram essas sugestões.

Da mesma forma, o uso de calço também promoveu reorientação segmentar da pelve e joelho. Resultados similares foram observados por Zabjek et al. (16) com a reorientação na inclinação da pelve em indivíduos com escoliose e obliquidade pélvica com o uso de palmilhas de diferentes alturas $(0,5$; 1,0 e $1,5 \mathrm{~cm}$ ). Portanto, o uso de calço ou palmila promove adaptações posturais agudas, alterarando a orientação da coluna vertebral e o posicionamento e orientação da pelve e da escápula.

Ainda, na situação com calços (baixo e alto) colocados sob o pé esquerdo, foi observada diferença entre os grupos para o ângulo postural toracolombar, sendo maior para o grupo experimental em relação ao grupo controle. As curvas maiores estavam do lado direito e o calço colocado sob o pé esquerdo mostrou maior influência nas curvas toracolombares; estas 
estão na transição das regiões torácica e lombar e, portanto, apresentam vértebras que acompanham a curva torácica e vértebras que acompanham a curva lombar. Isso indica que essa região poderia ser mais flexível porque seria menos afetada pela deformidade das vértebras no ápice da curva principal $(15,48)$.

De maneira geral, quando o calço é colocado sob um dos pés, podemos verificar que a maior influência ocorre nos ângulos posturais mais baixos, nas regiões toracolombar e lombar. As diferenças foram principalmente em relação aos grupos, mostrando que esses ângulos eram sempre maiores no grupo experimental. Esse resultado confirma que o calço tem efeito nas regiões mais baixas da coluna vertebral, menos afetada pela deformidade no ápice das curvas escolióticas e, portanto, com chances maiores de serem influenciadas pelo efeito corretivo dos calços na escoliose funcional. Os calços também influenciaram os ângulos segmentares mais baixos da pelve e do joelho. Esse resultado sugere que nas escolioses duplas qualquer alteração na base de apoio que modifica a simetria do tronco pode provocar reorganização das estruturas e busca de um novo arranjo do equilíbrio ou ajuste compensatório nos outros segmentos do tronco.

Nos programas de reabilitação, a base fundamental são flexibilidade (49) e reajustes posturais para buscar o alinhamento da coluna vertebral e de todo o corpo.

Nesse estudo, os ajustes agudos do calço sobre a coluna vertebral, pelo rearranjo postural nas regiões mais baixas e sobre os segmentos corporais da pelve e joelhos, podem ser aplicados por um período prolongado com possibilidades de ocorrer adaptações em longo prazo (18), o que possibilitaria sua utilização como proposta de tratamento, durante a adolescência ou mesmo depois da maturidade óssea. No entanto, é preciso avaliar a influência do calço em tipos de curvas específicas, e assim propor um calço adequado para cada tipo de curva.

\section{Conclusão}

Os ajustes agudos do calço sobre a coluna vertebral pelo rearranjo postural ocorreram nas regiões mais baixas e sobre os segmentos corporais da pelve e joelhos. Claramente os resultados deste estudo indicaram que, mesmo nos casos com escoliose, há flexibilidade e adaptação; portanto, a reorganização postural e segmentar seria susceptível a essas manipulações.

\section{Referências}

1. Horak FB, Macpherson JM. Postural orientation and equilibrium. In: Rowell LB, Shepherd JT. Handbook of physiology. Section 12: Exercise: regulation and integration of multiple systems. New York: Oxford University Press; 1996.

2. Fortin C, Feldman DE, Cheriet F, Labelle H. Clinical methods for quantifying body segment posture: a literature review. Disabil Rehabil. 2011;33(5):367-83. doi:10.3109/09638288.2010.492066.

3. Yang Z, Xie Y, Li M. Three-dimensional spring model: a new hypothesis of pathogenesis of adolescent idiopathic scoliosis. Med Hypoth. 2009;73:709-13. doi:10.1016/j.mehy.2009.02.046.

4. Bruyneel A-V, Chavet P, Bollin G, Allard P, Mesure S. The influence of adolescent idiopathic scoliosis on the dynamic adaptive behaviour. Neur Lette. 2008;447:15863. doi:10.1016/j.neulet.2008.10.007.

5. Döhnert MB, Tomasi E. Validade da fotogrametria computadorizada na detecção de escoliose idiopática adolescente. Rev Bras Fisioter. 2008;12(4):290-97. doi:10.1590/S1413-35552008000400007.

6. Ferreira DMA, Defino HLA. Avaliação quantitativa da escoliose idiopática: concordância das mensurações da gibosidade e correlações com medidas radiológicas. Rev Bras Fisioter. 2001;5(2):73-86.

7. Driscoll M, Aubin C-E, Moreau A, Villemure I, Parent $\mathrm{S}$. The role of spinal concave-convex biases in the progression of idiopathic scoliosis. Eur Spine J. 2008;18:180-87. doi:10.1007/s00586-008-0862-z.

8. Busscher I, Wapstra FH, Veldhuizen AG. Predicting growth and curve progression in the individual patient with adolescent idiopathic scoliosis: design of a prospective longitudinal cohort study. BMC Musculoskelet Disord. 2010;11(93):1-9.

9. Stehbens WE. Pathogenesis of idiopathic scoliosis revisited. Exp Mol Pathol. 2003;74:49-60. doi:10.1016/ S0014-4800(03)80008-4.

10. Gruber AH, Busa MA, Gorton III GE, Richard Van Emmerik REA, Masso PD, Hamill J.

11. Time-to-contact and multiscale entropy identify differences in postural control in adolescent idiopathic scoliosis. Gait Posture. 2011;34:13-18. doi:10.1016/j. gaitpost.2011.02.015. 
12. Allard P, Chavet P, Barbier F, Gatto L, Labelle H, Sadeghi H. Effect of body morphology on standing balance in adolescent idiopathic scoliosis. Am J Phys Med Rehab. 2004;83(9):689-97. doi:10.1097/01. PHM.0000137344.95784.15.

13. Gauchard GC, Lascombes P, Kuhnast M, Perrin PP. Influence of different types of progressive idiopathic scoliosis on static and dynamic control. Spine. 2001;26(9):1052-58. doi:10.1097/ 00007632-200105010-00014.

14. Bruyneel A-V, Chavet P, Bollini G, Allard P, Berton E, Mesure S. Dynamical asymmetries in idiopathic scoliosis during forward and lateral initiation step. Eur Spine J. 2009;18:188-95. doi:10.1007/s00586-008-0864-x.

15. Bruyneel A-V, Chavet P, Bollini G, Allard P, Berton E, Mesure S. Lateral steps reveal adaptive biomechanical strategies in adolescent idiopathic scoliosis. Ann Readapt Med Phys. 2008;51:630-64. doi:10.1016/j. annrmp.2008.05.004.

16. Hawes MC, O'Brien JP. The transformation of spinal curvature into spinal deformity pathological processes and implications for treatment. Scoliosis. 2006;1(3) [cited 2007 Jul. 4]. Available from: http:// www.scoliosisjournal.com/content/1/1/3.

17. Zabjek KF, Leroux MA, Coillard C, Martinez X, Griffet J, Simard G, et al. Acute postural adaptations induced by a shoe lift in idiopathic scoliosis patients. Eur Spine J. 2001;10:107-113. doi:10.1007/s005860000244.

18. Nault ML, Allard P, Hinse S, Le Blanc R, Caron O, Labelle $\mathrm{H}$, et al. Relations between standing stability and posture parameters in adolescent idiopathic scoliosis. Spine. 2002;27(17):1911-17. doi:10.1097/ 00007632-200209010-00018.

19. Beaudoin L, Zabjek KF, Leroux MA, Collard C, Rivard $\mathrm{CH}$. Acute systematic and variable postural adaptations induced by orthopaedic shoe lift in control subjects. Eur Spine J. 1999;8:40-45. doi:10.1007/s005860050125.

20. Nakajima K, Kakihana W, Nakagawa T, Mitomi H, Hikita A, Suzuki R, et al. Addition of an arch support improves the biomechanical effect of a laterally wedged insole. Gait Posture. 2009;29:208-13. doi:10.1016/j. gaitpost.2008.08.007.

21. Maruyama T, Kitagawa T, Takeshita K, Mochizuki K, Nakamura K. Conservative treatment for adolescent idiopathic scoliosis: can it reduce the incidence of surgical treatment. Ped Rehab. 2003;6(3-4):215-19.
22. Irvin RE. Reduction of lumbar scoliosis by use of a heel lift to level the sacral base. J Am Osteopath Assoc. 1991;91(1):34-44.

23. Fairbank J. Historical perspective. Willian Adams, the forward bending test, and the spine of Gideon Algernon Mantell. Spine. 2004;29(17):1953-55. doi:10.1097/01.brs.0000137072.41425.ec.

24. Engsberg JR, Lenke LG, Reitenbach AK, Hollander KW, Bridwell KH, Blanke K. Prospective evaluation of trunk range of motion in adolescents with idiopathic scoliosis undergoing spinal fusion surgery. Spine. 2002;27(12):1346-54. doi:10.1097/ 00007632-200206150-00018.

25. Feipel V, Aubin C.-E, Ciolofan OC, Beauséjour M, Labelle H, Mathieu PA. Electromyogram and kinematic analysis of lateral bending in idiopathic scoliosis patients. Med Biol Eng Comput. 2002;40:497-505. doi:10.1007/BF02345446.

26. Ferreira DMA, Carnielli AL, Ninello DA, Pachioni CAS, Fregonesi CEPT, Camargo MR. A influência do movimento de inclinação lateral na cifose torácica e na lordose lombar de indivíduos com escoliose idiopática. Ter Man. 2010;8(38):325-31.

27. Comerlato T. Avaliação da postura corporal estática no plano frontal a partir de imagem digital. [dissertação]. Porto Alegre: Escola de Educação Física, Universidade Federal do Rio Grande do Sul; 2007.

28. Ferreira DMA, Barela JA. Análise da influência do calço e do movimento de inclinação lateral da coluna vertebral em indivíduos com escoliose idiopática [tese]. Rio Claro: Departamento de Educação Física, Universidade Estadual Paulista, 2009.

29. Gonzalez LJ, Sreenivasan SV, Jensen JL. A procedure to determine equilibrium postural configurations for arbitrary locations of the feet. J Biomech Eng. 1999;121:644-49. doi:10.1115/1.2800867.

30. Ferreira DMA, Fernandes CG, Camargo MR, Pachioni CAS, Fregonesi CEPT, Faria CRS. Avaliação da coluna vertebral: relação entre gibosidade e curvas sagitais por método não-invasivo. Rev Bras Cineantropom Desempenho Hum. 2010;12(4):282-89.

31. Hoikka V, Ylioski M, Tallroth K. Leg-length inequality has poor correlation with lumbar scoliosis. Arch Orthop Trauma Surg. 1989;108:173-75. doi:10.1007/ BF00934262. 
32. Winter DA. Biomechanics and motor control of human movement. 4. ed. New York: John Wiley \& Sons, Inc; 2009. doi:10.1002/9780470549148.

33. Gram MC, Hasan Z. The spinal curve in standing and sitting postures in children with idiopathic scoliosis. Spine. 1999;24(2):169-77. doi:10.1097/ 00007632-199901150-00019.

34. Döhnert MB, Tomasi E. Validade da fotogrametria computadorizada na detecção de escoliose idiopática adolescente. Rev Bras Fisioter. 2008;12(4):290-97. doi:10.1590/S1413-35552008000400007.

35. Omkar SN, Kumar M, Mudigere D. Postural assessment of arbitrarily taken portrait and profile photographs using ImageJ. J Bodywork Move Ther. 2007;11:231-37. doi:10.1016/j.jbmt.2006.12.003.

36. Pereira OS. A utilização da análise computadorizada como método de avaliação das alterações posturais: um estudo preliminar. Fisioter Mov. 2003;16:17-25.

37. Zabjek KF, Leroux MA, Coillard C, Rivard C-H, Prince F. Evaluation of segmental postural characteristics during quiet standing in control and idiopathic scoliosis patients. Clin Biomech. 2005;20:483-90. doi:10.1016/j.clinbiomech.2005.01.003.

38. Ovadia D, Bar-On E, Fragnière B, Rigo M, Dickman D, Leitner J, et al. Radiation-free quantitative assessment of scoliosis: a multi center prospective study. Eur Spine J. 2007;16:97-105. doi:10.1007/s00586-006-0118-8.

39. Zubovic A, Davies N, Berriman F, Quraishi N, Lavy C, Bowden G, et al. New method of scoliosis deformity assessment ISIS- 2 system. Spine J. Proceedings of the NASS $23^{\text {rd }}$ Annual Meeting. 2008;8:29S.

40. Hackenberg L, Hierholzer E, Bullmann V, Liljenqvist U, Gotze C. Rasterstereographic analysis of axial back surface rotation in standing versus forward bending posture in idiopathic scoliosis. Eur Spine J. 2006;15(7):114449. doi:10.1007/s00586-005-0057-9.

41. Ferreira DMA, Suguikawa TR, Pachioni CAS, Fregonesi CEPT, Camargo MR. Rastreamento escolar da escoliose: medida para o diagnóstico precoce. Rev Bras Crescimento Desenvolv Hum. 2009;19(3):357-68.

42. Santos LM, Souza TP, Crescentini MCV, Poletto PR, Gotfryd AO, Yi LC. Avaliação postural por fotogrametria em pacientes com escoliose idiopática submetidos à artrodese: estudo piloto. Fisioter Mov. 2012;25(1):16573. doi:10.1590/S0103-51502012000100016.
43. Beaulieu M, Toulotte C, Gatto L, Rivard CH, Teasdale N, Simoneau M, et al. Postural imbalance in non-treated adolescent idiopathic scoliosis at different periods of progression. Eur Spine J. 2009;18:38-44. doi:10.1007/ s00586-008-0831-6.

44. Chow DHK, Kwok MLY, Cheng JCY, Lao MLM, Holmes $\mathrm{AD}$, Au-Yang A, et al. The effect of backpack weight on the standing of schoolgirls with adolescent idiopathic scoliosis and normal controls. Gait Posture. 2006;24:173-81. doi:10.1016/j.gaitpost.2005.08.007.

45. Dalleau G, Allard MS, Beaulieu M, Rivard CH, Allard P. Free moment contribution to quiet standing in able-bodied and scoliotic girls. Eur Spine J. 2007;16:159399. doi:10.1007/s00586-007-0404-0.

46. Byl NN, Gray JM. Complex balance reactions in different sensory conditions: adolescents with and without idiopathic scoliosis. J Orthop Res. 1993;11:215-27. doi:10.1002/jor.1100110209.

47. Byl NN, Holland S, Jurek A, Hu SS. Postural imbalance and vibratory sensitivity in patients with idiopathic scoliosis: implications for treatment. J Orthop Sports Phys Ther. 1997;26:60-68.

48. Herman R, Mixon J, Fisher A, Maulucci R, Stuyck J. Idiopathic scoliosis and the central nervous system: a motor control problem. Spine. 1985;10:1-14. doi:10.1097/00007632-198501000-00001.

49. Modi HN, Suh SW, Song HR, Yang JH, Kim HJ, Modi CH. Differential wedging of vertebral body and intervertebral disc in thoracic and lumbar spine in adolescent idiopathic scoliosis - a cross sectional study in 150 patients [serial on the internet]. Scoliosis. 2008; 3(11) [cited 23 Mar. 2009]. Available from: http://www. scoliosisjournal.com/content/3/1/11.

Recebido: $16 / 05 / 2012$

Received: 05/16/2012

Aprovado: 25/11/2012 Approved: $11 / 25 / 2012$ 